\title{
Review
}

\section{The population genetics of beneficial mutations}

\author{
H. Allen Orr* \\ Department of Biology, University of Rochester, Rochester, NY 14627, USA
}

\begin{abstract}
The population genetic study of advantageous mutations has lagged behind that of deleterious and neutral mutations. But over the past two decades, a number of significant developments, both theoretical and empirical, have occurred. Here, I review two of these developments: the attempt to determine the distribution of fitness effects among beneficial mutations and the attempt to determine their average dominance. Considering both theory and data, I conclude that, while considerable theoretical progress has been made, we still lack sufficient data to draw confident conclusions about the distribution of effects or the dominance of beneficial mutations.
\end{abstract}

Keywords: adaptation; beneficial mutations; dominance; extreme value theory; $\mathrm{X}$ chromosome

\section{INTRODUCTION AND HISTORY}

The history of population genetics, especially theoretical population genetics, presents a paradox. Although population genetics arose from the fusion of Darwin's theory of adaptation by natural selection with Mendel's theory of inheritance, the field has been concerned historically far more with deleterious and, later, with neutral mutations than with beneficial ones. The paradox has, of course, a simple solution: population geneticists were looking under the lamp post. In particular, mutations having negative or negligible effects on fitness are more common and thus easily studied, while those having positive effects on fitness are far rarer and thus studied only with difficulty.

Though understandable, the neglect of beneficial mutations was unfortunate for two reasons. First and obviously, without considering positive selection, population genetics could make no real contribution to the study of adaptation. Second and less obviously, the key result on beneficial mutations to emerge from the early years of theoretical population genetics revealed that the mathematics of positive selection were deeply counterintuitive, and so could be neglected only with risk.

In particular, Haldane (1927) considered the fate of a unique mutation having beneficial effect $s$ in heterozygotes. He asked, in effect, whether Darwin's directional force of natural selection could overcome the noise introduced partly (though not entirely) by Mendel's law of segregation: a heterozygote that carries a new beneficial mutation enjoys an enhanced fitness of $1+s$ but, because of segregation, might pass on no copies of the beneficial allele to progeny. Using branching process theory, Haldane found that

*aorr@mail.rochester.edu

One contribution of 16 to a Theme Issue 'The population genetics of mutations: good, bad and indifferent' dedicated to Brian Charlesworth on his 65 th birthday. beneficial mutations become fixed only $p_{\text {fix }} \approx 2 s$ of the time. A new mutation that improves fitness by $s=0.001$ must therefore appear by mutation on average $1 / p_{\text {fix }}=500$ times in the population before being fixed by natural selection-a surprising finding.

For nearly half a century after the publication of Haldane's paper, few results on positive selection of major significance appeared in the literature. (The main exception involved the debate over the cost of selection, or substitution load, a debate that ultimately included Haldane (1957), Maynard Smith (1968), Crow (1970), Felsenstein (1971) and Kimura \& Ohta (1971), among others.)

But in the 1970s and 1980s, a set of papers appeared that focused attention on the population genetics of beneficial mutations. In retrospect, several developments were particularly significant. First, Maynard Smith \& Haigh (1974) analysed the effects of hitchhiking on levels of neutral polymorphism at linked sites. Second, Gillespie (1983, 1984) and Kauffman \& Levin (1987) introduced models of adaptation in discrete (DNA) sequence spaces. Third, Charlesworth et al. (1987) analysed the effects of dominance and linkage group (e.g. X-linkage versus autosomal linkage) on rates of molecular evolution under positive selection. While the theory of hitchhiking received most attention from evolutionary geneticists, a good case could be made that work on the adaptation of DNA sequences and on the effects of dominance and linkage group represents a more direct contribution to the population genetics of adaptation. (Hitchhiking is concerned with the consequences of adaptive evolution, while the latter two developments consider adaptive evolution per se.) In any case, it seems clear that these several developments played important parts in the recent, and sustained, interest in the population genetics of beneficial mutations.

Here, I briefly review two problems that have been at the centre of recent efforts. In particular, I ask: 
What is the distribution of fitness effects among beneficial mutations? And, how dominant are beneficial mutations? In each case, I consider both theory and experiment.

\section{THE DISTRIBUTION OF FITNESS EFFECTS AMONG BENEFICIAL MUTATIONS: THEORY}

Just as work early in the history of population genetics attempted to estimate the distribution of fitness effects among deleterious mutations-this represented the focus of much of Dobzhansky's efforts in his Genetics of natural populations series (Lewontin et al. 1981)so recent work has attempted to estimate the distribution of fitness effects among beneficial mutations. As noted above, however, there is a large difference between the two cases: the former involves mutations that are reasonably common and that may segregate at non-trivial frequencies in nature, while the latter involves mutations that are far rarer and that are therefore not easily observed in nature. Estimating even the mean effect, much less the distribution of effects, of beneficial mutations is thus extraordinarily difficult.

Fortunately, however, a shortcut may be possible. Contrary to intuition, the very rarity of beneficial mutations may allow reasonable guesses about the distribution of beneficial fitness effects. In particular, the combined facts that (i) beneficial mutations are rare; and (ii) the wild-type allele is usually of high fitness, mean that extreme value theory (EVT) might provide a clue about the distribution of fitness effects.

EVT considers the behaviour of draws from the tails of probability distributions. While classical EVT was developed largely in the context of flood prediction (e.g. the height of 100-year floods; Gumbel 1958), it has been applied subsequently to problems in physics, chemistry and especially mathematical finance (as investors are interested in the probabilities of unusually large swings in stock prices). While Ewens (1970) and Kimura \& Ohta (1971, p. 80) used EVT in population-genetic contexts, Gillespie (1983, 1984, 1991) first realized that the theory might allow predictions about the distribution of fitness effects among beneficial mutations.

In the scenario usually considered, selection is strong $(|N s|>1$, where $N$ is population size and $s$ is a selection coefficient) and mutation is weak ( $N u \ll 1$, where $u$ is the per site mutation rate). (Throughout I will assume, for convenience, that effective and census population sizes are equal.) Under these so-called strong-selection weak-mutation conditions, the population is essentially made up of a single wild-type DNA sequence. Given a sequence that is $L$ base pairs long, the wild-type recurrently mutates to $m=3 L$ different mutant sequences. (Because mutation is rare, double- and higher-order mutations can be neglected.) Each of these sequences, including the wild-type, is assigned a fitness from some distribution. The key point, however, is that this overall distribution of fitness is unknown. Despite this, we do know two things. First, the wild-type allele is highly fit; indeed it is fitter than all of its $m$ mutant 'neighbour' sequences (this is why it is wild-type). Second, any beneficial mutations would be even fitter and so would fall even farther out in the tail of the fitness distribution. (We assume for now that this tail falls off in some 'ordinary' smooth way; see below.) At some point in time, the environment changes and the wild-type allele slips slightly in fitness and one or more of the $m$ mutations becomes beneficial. The question is: what is the size of the fitness gap between the wild-type and a beneficial sequence?

To answer this, Gillespie assumed that only one beneficial mutation is available. Taking advantage of an obscure part of EVT concerned with 'extreme spacings,' he showed that, more or less independently of the shape of the unknown overall distribution of fitness, this fitness gap-the fitness effects of new beneficial mutations-is exponentially distributed. This result was later generalized by Orr (2003) to any modest number of beneficial mutations (i.e. the wild-type sequence might mutate to 5 or 10 or so different beneficial mutations). Mutation should thus often yield beneficial alleles of small effect and rarely yield those of large effect. In retrospect, it is clear that this exponential distribution of beneficial effects is a simple consequence of a well-known result from so-called peaks-over-threshold models in EVT (Leadbetter et al. 1983). A large set of results, concerning both the first substitution during adaptation and the properties of entire adaptive walks to local optima rest on this result (Orr 2002, 2004, 2005; Rokyta et al. 2005).

It is worth recalling that this exponential finding depends on two assumptions: that the wild-type is highly fit and that the overall fitness distribution has a tail that falls off in some 'ordinary' way. While the first assumption seems reasonable, the second can be, and has been, questioned. In particular, both Gillespie and Orr assumed that the distribution of fitness, while unknown, falls into the 'Gumbel domain of attraction', a group of probability distributions that includes most ordinary distributions (e.g. normal, gamma, exponential, logistic). But this need not be true (Leadbetter et al. 1983; and in mathematical finance is known not to be true-price changes in equity markets appear to be drawn from distributional tails that are heavier than Gumbel). The point is that EVT reveals that two other domains of attraction, while more exotic, are possible: the Fréchet and Weibull domains (Leadbetter et al. 1983; Orr 2005). In the Fréchet domain, tails are heavier than the exponential ones characterizing the Gumbel domain, i.e. they fall off more slowly. Distributions belonging to the Fréchet domain are exotic in the sense that they lack higher or even all moments. In the Weibull domain, tails are truncated, as with a uniform distribution. (To make matters complicated, the Gumbel domain also includes some truncated distributions.).

Recently, Joyce et al. (2008) generalized the theory of beneficial mutations to allow the distribution of fitnesses to fall into any of the three domains of attraction of tail behaviour. In particular, they analysed the generalized Pareto distribution (GPD), which describes the distribution of values above a high threshold (e.g. wild-type fitness). The probability 
density of the GPD is given by

$f(x)=\frac{1}{\tau}\left(1+\frac{\kappa x}{\tau}\right)^{-(\kappa+1) / \kappa}$,

where $\tau$ is a scale parameter and $\kappa$ determines shape. By tuning the parameter $\kappa$, equation (2.1) yields a distribution that falls into any of the three extreme value domains of attraction: if $\kappa=0$ (or, more exactly, as $\kappa \rightarrow 0$ ) the distribution falls into the Gumbel domain; if $\kappa>0$, it falls into the Fréchet domain; and if $\kappa<0$, it falls into the Weibull domain (see Joyce et al. (2008) for details). By analysing adaptation using the GPD, Joyce et al. arrived at several interesting results. First, if departures from the Gumbel domain are mild, i.e. $-1 / 2<\kappa<1 / 2$, traditional results from the genetics of adaptation concerning single substitutions - results that rest on Gumbel theory-remain approximately correct. The reason, roughly put, is that the distribution of fitness effects among new beneficial mutations remains sufficiently 'exponential-ish' that traditional theory holds. This is an important, and reassuring, result. Second, under the same conditions, results for entire adaptive walks involving several to many adaptive substitutions can show substantial departures from traditional (Gumbel-based) genetics of adaptation. Third, as departures from the Gumbel domain grow larger, traditional theory typically does not hold. Though it appears biologically impossible to obtain deviations from Gumbel theory when the distribution of fitness effects belongs to the Fréchet domain (see Joyce et al. (2008) for more on this result), deviations from Gumbel theory can occur when the distribution of fitness effects is truncated and belongs to the Weibull domain.

\section{THE DISTRIBUTION OF FITNESS EFFECTS AMONG BENEFICIAL MUTATIONS: DATA}

The possibility that the distribution of fitness effects might often be approximately exponential has generated considerable interest among experimentalists. Indeed, several have been motivated to attempt the daunting task of estimating the distribution of effects among beneficial mutations in actual organisms. While quantitative geneticists have shown some interest in back-calculating the desired distribution from quantitative trait locus data, here I discuss data from microbial experimental evolution studies as these analyses provide higher resolution data. In these studies, experimentalists typically allow a clonal population of microbes to adapt to a novel environmental challenge and then attempt to isolate new mutations that are advantageous under these changed conditions. The fitness effects of these mutations can then be assessed, e.g. by competing the mutations against the ancestral microbe under the new conditions.

It should be emphasized that the distribution we hope to characterize experimentally is that among new beneficial mutations, not among those that are substituted during adaptation. The latter distribution can differ from the former for two reasons. First, even when beneficial mutations are rare, the distribution of fitness effects among substituted mutations is affected by probabilities of fixation (Kimura 1983).
Second, if beneficial mutations are more commonas they could be in artificial or extreme laboratory conditions - clonal interference among multiple beneficial mutations can further distort the distribution of effects among substituted mutations, as larger-effect mutations competitively displace smaller-effect ones (Gerrish \& Lenski 1998). To make matters worse, the distribution of effects among substituted mutations given clonal interference also falls into the Gumbel domain (Rozen et al. 2002). Experimentalists must thus attempt to sidestep these problems, estimating the desired distribution of effects among newly arising mutations.

Unfortunately, the data available thus far from the relevant experiments are mixed. Several experiments have found that the distribution of beneficial effects among new mutations is at least roughly exponential. Kassen \& Bataillon (2006), for example, measured the fitness effects of newly arising beneficial mutations in the bacterium Pseudomonas fluorescens. Although they were unable to identify individual mutations at the DNA sequence level (and so were unable to determine the number of distinct mutations sampled), they could not reject the null hypothesis of an exponential distribution of beneficial effects. Similarly, Sanjuan et al. (2004) measured the fitness effects of new beneficial mutations in vesicular stomatitis virus. Although few distinct mutations were obtained, they concluded that the distribution of beneficial effects was gamma, with a shape parameter close to (but statistically distinguishable from) exponential. (The gamma distribution belongs to the Gumbel domain.) In both experiments, beneficial mutations of small effect were more common than those of large effect (similarly, see Imhof \& Schlotterer 2001; Perfeito et al. 2007).

These results contrast, however, with those obtained in recent work with the bacteriophages ID1 1 and $\phi 6$. Rokyta et al. (2008) isolated and sequenced new mutations that adapt the DNA phage ID11 to increased growth on $E$. coli host or that adapt the RNA phage $\phi 6$ to use the non-permissive host Pseudomonas syringae. Employing a statistical (likelihood ratio test) approach developed by Beisel et al. (2007) — which explicitly tests whether the distribution of beneficial effects belongs to the Gumbel domain $(\kappa=0)$ or to an alternative one $(\kappa \neq 0)$ they found that the distribution of effects was not Gumbel. Instead, the distribution belonged to the Weibull domain, i.e. was truncated at the top. Even more surprisingly, the distribution of beneficial effects roughly resembled a uniform distribution $(\kappa \approx-1)$ : large-effect beneficial mutations appeared as often as small-effect ones, contrary to the assumption of most evolutionary biologists.

Given these mixed results, it is difficult, at present, to draw firm conclusions. As each experiment measures (with error) fitness in one taxon in one environment and generally from one wild-type sequence, we cannot yet have confidence in any generalities - or the lack thereof. It may be worth noting, however, one possible explanation of the difference between the exponential-like results seen in some experiments and the uniform-like ones seen by 
Rokyta et al. (2008). When a mutation of given size is allowed to have random direction in Fisher's geometric model of adaptation, the distribution of fitness effects among beneficial mutations is more uniform-like at low dimensions (i.e. few characters) and more exponential-like at high dimensions (many characters). In the Fisherian limit of infinitely many characters, the distribution falls into the Gumbel domain and becomes essentially exponential (Orr 2006; Martin \& Lenormand 2008). This conclusion makes good intuitive sense: it is easier for a large mutation to be beneficial when it can affect only a few characters, not many. Though wholly speculative, it is possible that this finding has some bearing on the bacteriophage data discussed above as phages are presumably of low dimensionality.

\section{THE DOMINANCE OF BENEFICIAL MUTATIONS: THEORY}

Just as we can ask about the additive effects on fitness of beneficial mutations, so we can ask about their dominance. In diploids, are most beneficial mutations recessive or dominant for fitness? Once again, the rarity of beneficial mutations makes it difficult to answer this question directly.

Nearly all modern literature on the dominance of beneficial mutations traces to a seminal paper by Charlesworth et al. (1987). In this paper, Charlesworth and colleagues presented a general analysis of the evolution of genes that reside in different regions of the genome and that, consequently, show different patterns of inheritance. In particular, they considered the evolution of sex chromosomes versus autosomes, investigating both single-locus evolution and evolution at a quantitative character that has experienced a shift in optimum. For simplicity-and because it became the focus of subsequent research-I consider here only single-locus results. As we will see, the contrast between rates of evolution at X-linked versus autosomal genes often depends on the dominance of beneficial mutations.

As Charlesworth et al. note, almost no theoretical analyses of long-term evolution on the sex chromosomes versus autosomes had appeared since Haldane's $(1924,1926)$ original studies. The problem was clearly not the mathematical difficulty of the problem for, as Charlesworth et al. again note, the required model is simple. Indeed under the simplest possible model, long-term evolution is the product of two quantities: the number of beneficial mutations that appear per generation and the probability of their fixation (Haldane 1927; Kimura \& Ohta 1971). In the case of an autosomal locus, $2 \mathrm{Nu}$ new unique mutations appear per generation and, given reasonably weak selection and equal selection in the two sexes, each beneficial mutation enjoys a probability of fixation of $2 h s$, where $h$ is the dominance coefficient ( $h=0$ means the beneficial mutation is completely recessive, $h=\frac{1}{2}$ means it is additive, and $h=1$ means it is completely dominant). This result, a branching process approximation, is reasonably accurate unless $h$ is close to zero (Moran 1962). Thus, the rate of adaptive molecular evolution at an autosomal locus is $k_{A} \approx 4$ Nuhs.

Turning to X-linked loci, both the number of beneficial mutations that appear per generation and their probability of fixation differ from the autosomal case. Considering mammal- or Drosophila-like species in which females carry two $\mathrm{X}$ chromosomes and males one, three-fourths as many $\mathrm{X}$ chromosomes as autosomes reside in a population; thus $3 N u / 2$ unique mutations appear per generation, where we assume that $u$ is the same at $\mathrm{X}$-linked and autosomal loci. With weak selection, it can be shown that the probability of fixation of an X-linked mutation approximately equals the weighted average of the relevant probabilities for female-limited and malelimited mutations (Nagylaki 1979): $2 h s$ and $2 s$, respectively, where the latter probability reflects the haploid-like probability of fixation of a hemizygous mutation. (We assume dosage compensation such that a single dose of the mutation in males provides the same fitness advantage as two doses in females.) As two-thirds of all X-linked mutations arise in females and one-third in males, the average probability of fixation becomes $2 s(1+2 h) / 3$. Thus the rate of adaptive molecular evolution of an X-liked locus is $k_{X} \approx N u s(1+2 h)$.

Finally, then, we see that the ratio of substitution rates on autosomes to $\mathrm{X}$ chromosomes is

$$
R \approx \frac{4 h}{1+2 h}
$$

Importantly, this ratio depends only on the dominance of beneficial mutations.

Charlesworth et al. generalized the above approach to allow for Y-linkage, selection in one sex only, with or without dosage compensation (including marsupiallike dosage compensation), underdominance, and antagonistic selection between the two sexes. In addition, they considered evolution due to the fixation of slightly deleterious mutations. (In much more recent work, Vicoso \& Charlesworth (2009) extend this theory to allow ratios of effective population sizes on the $\mathrm{X}$ versus autosomes that deviate from 3/4 because of, e.g. male-male competition for mates. I do not consider this complication here.)

The resulting body of theory has experienced a somewhat surprising history. Though presented initially in the contexts of chromosomal evolution and speciation-much of Charlesworth et al.'s discussion was given over to patterns characterizing the genetics of postzygotic isolation, in particular Haldane's rule and the large effect of the X chromosome on hybrid sterility and inviability (Coyne \& Orr 1989) - the theory has, in more recent years, played an important part in the interpretation of DNA sequence data, originally from small sets of genes and, more recently, from whole genomes. Equation (4.1) has been the focus of this attention. In particular, this equation shows that, if long-term evolution reflects positive natural selection and all else is equal, patterns of species divergence across the genome depend on the dominance of beneficial mutations. If beneficial mutations are additive $\left(h=\frac{1}{2}\right), R=1$ and autosomal 
and $\mathrm{X}$-linked sites evolve at the same rate. If beneficial mutations are partially recessive $\left(h<\frac{1}{2}\right), R<1$ and $\mathrm{X}$-linked sites evolve faster than autosomal ones, reflecting the full expression of favourable effects of $\mathrm{X}$-linked mutations in hemizygous males and thus more efficient positive natural selection. And if beneficial mutations are partially dominant $\left(h>\frac{1}{2}\right)$, $R>1$ and $\mathrm{X}$-linked sites evolve more slowly than autosomal ones. (In all cases, we can now speak of the average dominance of beneficial mutations: as substitution rates are approximately linear in $h$, expected substitution rates depend linearly on mean $h$. Equation (4.1) can, therefore, be interpreted as a ratio of expectations.).

The key question is therefore: is the rate of substitution at X-linked sites-especially non-synonymous ones, where positive selection is more probablefaster or slower than that at autosomal sites? Expressed in the language that has come to describe this problem, is there 'faster- $\mathrm{X}$ ' evolution?

\section{THE DOMINANCE OF BENEFICIAL MUTATIONS: DATA}

The data that bear on this question are now both voluminous and complex and I can do little more than indicate the rough outlines of recent findings, while pointing to other, more detailed, discussions.

Betancourt et al. (2002) provided one of the earliest studies to compare species divergence at many $\mathrm{X}$ linked versus autosomal genes. Analysing DNA sequence data from both Drosophila melanogaster and D. simulans at over 200 loci, they found no evidence of faster-X evolution. In particular, they found, among other things, that the ratio of non-synonymous site to synonymous site divergence-a ratio that, when greater than unity, indicates a history of positive natural selection-was no higher at X-linked than at autosomal genes. This result would seem to imply that the average beneficial mutation has nearly additive $\left(h=\frac{1}{2}\right.$ ) effects on fitness. This conclusion, however, rests upon an assumption of ceteris paribus, i.e. $\mathrm{X}$-linked and autosomal genes are exchangeable biologically and, in particular, show similar levels of functional constraint. While reasonable, this need not be true.

Counterman et al. (2004) identified a way to circumvent this concern. They analysed the same set of loci in two different species pairs. In one pair (D. melanogaster and D. simulans) the set of loci is autosomal (chromosome arm $3 L$ ), while in the other pair of species (D. pseudoobscura and D. miranda), the same set of loci is $\mathrm{X}$-linked (chromosome arm $X R$ ), reflecting an ancient fusion of chromosomal arms in the obscura group. Their statistical contrasts revealed somewhat faster non-synonymous site evolution when loci are X-linked than autosomal, although the effect was weak. While this result tentatively implied that the average beneficial mutation has partially recessive effects on fitness $\left(h<\frac{1}{2}\right)$, a similar analysis using a larger dataset did not reach the same conclusion. In particular, Thornton et al. (2006) studied over 200 gene fragments that are autosomal in D. melanogaster and D. yakuba but X-linked in D. pseudoobscura and
D. miranda. They found no evidence of faster-X evolution, consistent with the hypothesis that the average beneficial mutation has nearly additive effects on fitness.

Unfortunately - as both Vicoso \& Charlesworth (2006) and Presgraves (2008) emphasize in their authoritative reviews of this literature-this frustrating ambiguity in the outcomes of empirical tests of faster-X evolution has continued. Presgraves (2008) exhaustively reviewed the tests performed to date in Drosophila and identified 10 studies that found roughly equal rates of $\mathrm{X}$-linked versus autosomal evolution and 12 studies that found somewhat faster X-linked than autosomal evolution. These studies differed in many ways, including the species considered, the number of genes analysed, the nature of the data used to test faster-X evolution (species divergence data only versus divergence and polymorphism data versus amino acid sequence data only), and whether genes were binned by male versus female versus both-sex expression patterns (Baines et al. (2008) find that genes with male-biased expressed show greater faster-X evolution than do those with both-sex or female-limited expression). Looking across these heterogeneous studies, Presgraves concluded that there is some evidence of weakly elevated rates of substitution on the $\mathrm{X}$ chromosomes of some fly lineages. This idea is further supported by work in mammals, e.g. human-chimpanzee contrasts (reviewed in Vicoso \& Charlesworth (2006)) and, interestingly, in birds, where males carry two Z ('X') chromosomes and females only one: Mank et al. (2007) found a small but statistically significant faster- $Z$ effect when analysing sequence divergence at $172 \mathrm{Z}$-linked and nearly 5000 autosomal genes between the chicken and zebra finch.

Collectively, these findings are consistent with somewhat faster $\mathrm{X}$ evolution in some species, though not all. The difficult question is why such mixed results arise. One possibility is that taxa-or the genes sampled in particular studies of taxa-differ in the proportion of non-synonymous sites whose evolution is actually driven by strong positive selection (Vicoso \& Charlesworth 2006; Vicoso et al. 2008), or, more oddly, in the mean dominance of beneficial mutations, or, perhaps, in the proportion of X-linked genes that show male-biased expression patterns. Similarly, as Presgraves (2008) noted, lineages may differ in mutational properties affecting $\mathrm{X}$ versus autosomal evolution. (One such complication can, however, be ruled out: Vicoso \& Charlesworth (2009) show that higher rates of mutation in the male than female germline do not affect tests of faster-X evolution so long as non-synonymous divergence is normalized by synonymous divergence.) Finally, it must be noted that the theory considered above might be irrelevant, or even misleading, if much long-term adaptive molecular evolution involves the fixation not of new mutations but of alleles from the standing genetic variation that become favourable under changed conditions (Orr \& Betancourt 2001; Vicoso \& Charlesworth 2006).

Given these (and other) uncertainties, Vicoso \& Charlesworth (2006) conclude that 'no unambiguous 
conclusions concerning causality' can be drawn; in particular, no firm conclusions about the dominance of beneficial mutations currently appear possible from molecular evolutionary data. It may be that firm conclusions will be possible only when genome sequence data are available from many more taxa, potentially allowing a clearer mapping between the biology of the various species surveyed and whether they show faster-X evolution.

Alternatively, it may well be that radically different-and more direct-approaches are needed, involving, say, contrasts between the dominance of derived adaptive states in inbreeding versus outbreeding plants (Charlesworth 1992) or between rates of adaptive substitution in haploid versus diploid strains of microbes under novel laboratory conditions (Paquin \& Adams 1983; Zeyl et al. 2003). In both cases, population-genetic theory allows simple predictions about experimental outcomes as a function of the dominance of beneficial mutations.

\section{CONCLUDING REMARKS}

Although the population genetics of beneficial mutations was long ignored, progress has been made over the past several decades. This progress has, however, been uneven. As often in the history of population genetics, the theoretical literature is farther along - and is far less ambiguous - than the empirical literature. Here, I have discussed two developments. First, theory has allowed reasonable predictions about the shape of the distribution of fitness effects among beneficial mutations. Second, calculations on substitution rates under positive natural selection in different regions of the genome have allowed predictions of the effects of the dominance of beneficial mutations on adaptive molecular evolution.

In both cases, the data have yet to catch up with the theory. Importantly, however, in both cases theory has at least allowed questions to be framed with greater precision (e.g. is the distribution of beneficial fitness effects in the Gumbel domain?). There can also be little doubt that, in both cases, the articulation of theory motivated the beginnings of serious empirical work. Although it is clear that, at present, we require far more data than theory, advances in rapid whole genome sequencing technologies fortunately suggest that at least some of the requisite data might be obtained in the near future.

I thank Laurence Loewe and two anonymous reviewers for helpful comments on the manuscript. And I congratulate Brian Charlesworth on his many, and truly inspiring, accomplishments over the years; many more are surely on the way. H.A.O. is supported by National Institutes of Health grant GM51 932.

\section{REFERENCES}

Baines, J. F., Sawyer, S. A., Hartl, D. L. \& Parsch, J. 2008 Effects of X-linkage and sex-biased gene expression on the rates of adaptive protein evolution in Drosophila. Mol. Biol. Evol. 25, 1639-1650. (doi:10.1093/molbev/ msn111)

Beisel, C. J., Rokyta, D. R., Wichman, H. A. \& Joyce, P. 2007 Testing the extreme value domain of attraction for distributions of beneficial fitness effects. Genetics 176, 2441-2449. (doi:10.1534/genetics.106.068585)

Betancourt, A. J., Presgraves, D. C. \& Swanson, W. J. 2002 A test for faster X evolution in Drosophila. Mol. Biol. Evol. 19, 1816-1819.

Charlesworth, B. 1992 Evolutionary rates in partially selffertilizing species. Am. Nat. 140, 126-148.

Charlesworth, B., Coyne, J. A. \& Barton, N. 1987 The relative rates of evolution of sex chromosomes and autosomes. Am. Nat. 130, 113-146.

Counterman, B. A., Ortiz-Barrientos, D. \& Noor, M. A. F. 2004 Using comparative genomic data to test for fast-X evolution. Evolution 53, 656-660.

Coyne, J. A. \& Orr, H. A. 1989 Two rules of speciation. In Speciation and its consequences (eds D. Otte \& J. Endler), pp. 180-207. Sunderland, MA: Sinauer Associates.

Crow, J. F. 1970 Genetic loads and the cost of natural selection. In Mathematical models in population genetics (ed. K.-I. Kojima), pp. 128-177. Berlin, Germany: Springer.

Ewens, W. J. 1970 Remarks on the substitution load. Theor. Popul. Biol. 1, 129-139. (doi:10.1016/00405809(70)90031-6)

Felsenstein, J. 1971 On the biological significance of the cost of gene substitution. Am. Nat. 105, 169-184.

Gerrish, P. J. R. \& Lenski, E. 1998 The fate of competing beneficial mutations in an asexual population. Genetica 102/103, 127-144. (doi:10.1023/A:1017067816551)

Gillespie, J. H. 1983 A simple stochastic gene substitution model. Theor. Popul. Biol. 23, 202-215. (doi:10.1016/ 0040-5809(83)90014-X)

Gillespie, J. H. 1984 Molecular evolution over the mutational landscape. Evolution 38, 1116-1129. (doi:10. 2307/2408444)

Gillespie, J. H. 1991 The causes of molecular evolution. Oxford, UK: Oxford University Press.

Gumbel, E. J. 1958 Statistics of extremes. New York, NY: Columbia University Press.

Haldane, J. B. S. 1924 A mathematical theory of natural and artificial selection, Part I. Trans. Camb. Phil. Soc. 23, $19-41$.

Haldane, J. B. S. 1926 A mathematical theory of natural and artificial selection. Part III. Proc. Camb. Phil. Soc. 23, 363-372. (doi:10.1017/S0305004100015176)

Haldane, J. B. S. 1927 A mathematical theory of natural and artificial selection, Part V: Selection and mutation. Proc. Camb. Phil. Soc. 23, 838-844. (doi:10.1017/ S0305004100015644)

Haldane, J. B. S. 1957 The cost of natural selection. F. Genet. 55, 511-524. (doi:10.1007/BF02984069)

Imhof, M. \& Schlotterer, C. 2001 Fitness effects of advantageous mutations in evolving Escherichia coli populations. Proc. Natl Acad. Sci. USA 98, 1113-1117. (doi:10.1073/pnas.98.3.1113)

Joyce, P., Rokyta, D. R., Beisel, C. J. \& Orr, H. A. 2008 A general extreme value theory model for the adaptation of DNA sequences under strong selection and weak mutation. Genetics 180, 1627-1643. (doi:10.1534/ genetics.108.088716)

Kassen, R. \& Bataillon, T. 2006 Distribution of fitness effects among beneficial mutations before selection in experimental populations of bacteria. Nat. Genet. 38, 484-488. (doi:10.1038/ng1751)

Kauffman, S. \& Levin, S. 1987 Towards a general theory of adaptive walks on rugged landscapes. F. Theor. Biol. 128, 11-45. (doi:10.1016/S0022-5193(87)80029-2)

Kimura, M. 1983 The neutral theory of molecular evolution. Cambridge, UK: Cambridge University Press.

Kimura, M. \& Ohta, T. 1971 Theoretical aspects of population genetics. Princeton, NJ: Princeton University Press. 
Leadbetter, M. R., Lindgren, G. \& Rootzen, H. 1983 Extremes and related properties of random sequences and processes. New York, NY: Springer.

Lewontin, R. C., Moore, J. A., Provine, W. B. \& Wallace, E. B. 1981 Dobzhansky's genetics of natural populations I-XLIII. New York, NY: Columbia University Press.

Mank, J. L., Axelsson, E. \& Ellegren, H. 2007 Fast-X on the $\mathrm{Z}$ : rapid evolution of sex-linked genes in birds. Genome Res. 17, 618-624. (doi:10.1101/gr.6031907)

Martin, G. \& Lenormand, T. 2008 The distribution of beneficial and fixed mutation fitness effects close to an optimum. Genetics 179, 907-916. (doi:10.1534/genetics. 108.087122)

Maynard Smith, J. 1968 Haldane's dilemma and the rate of evolution. Nature 219, 1114-1116. (doi:10.1038/ 2191114a0)

Maynard Smith, J. \& Haigh, J. 1974 The hitch-hiking effect of a favourable gene. Genet. Res. 23, 23-25.

Moran, P. A. P. 1962 The statistical processes of evolutionary theory. Oxford, UK: Clarendon Press.

Nagylaki, T. 1979 Selection in dioecious populations. Ann. Hum. Genet. 43, 143-150. (doi:10.1111/j.14691809.1979.tb02007.x)

Orr, H. A. 2002 The population genetics of adaptation: the adaptation of DNA sequences. Evolution 56, 1317-1330.

Orr, H. A. 2003 The distribution of fitness effects among beneficial mutations. Genetics 163, 1519-1526.

Orr, H. A. 2004 The probability of parallel adaptation. Evolution 59, 216-220.

Orr, H. A. 2005 Genetic theories of adaptation: a brief history. Nat. Rev. Genet. 6, 119-127. (doi:10.1038/nrg1523)

Orr, H. A. 2006 The distribution of beneficial fitness effects among beneficial mutations in Fisher's geometric model of adaptation. F. Theor. Biol. 238, 279-285. (doi:10. 1016/j.jtbi.2005.05.001)

Orr, H. A. \& Betancourt, A. 2001 Haldane's sieve and adaptation from the standing genetic variation. Genetics 157, 875-884.

Paquin, C. \& Adams, J. 1983 Frequency of fixation of adaptive mutations is higher in evolving diploid than haploid yeast populations. Nature 302, 495-500. (doi:10.1038/ 302495a0)
Perfeito, L., Fernandes, L., Mota, C. \& Gordo, I. 2007 Adaptive mutations in bacteria: high rate and small effects. Science 317, 813-815. (doi:10.1126/science. 1142284)

Presgraves, D. C. 2008 Sex chromosome and speciation in Drosophila. Trends Genet. 24, 336-343. (doi:10.1016/j. tig.2008.04.007)

Rokyta, D. R., Joyce, P., Caudle, S. B. \& Wichman, H. A. 2005 An empirical test of the mutational landscape model of adaptation using a single-stranded DNA virus. Nat. Genet. 37, 441-444. (doi:10.1038/ng1535)

Rokyta, D. R., Beisel, C. J., Joyce, P., Ferris, M. T., Burch, C. L. \& Wichman, H. A. 2008 Beneficial effects are not exponential for two viruses. F. Mol. Evol. 67, 368-376. (doi:10.1007/s00239-008-9153-x)

Rozen, D. E., de Visser, J. A. \& Gerrish, P. J. 2002 Fitness effects of fixed beneficial mutations in microbial populations. Curr. Biol. 12, 1040-1045. (doi:10.1016/ S0960-9822(02)00896-5)

Sanjuan, R., Moya, A. \& Elena, S. F. 2004 The distribution of fitness effects caused by single-nucleotide substitutions in an RNA virus. Proc. Natl Acad. Sci. USA 101, 83968401. (doi:10.1073/pnas.0400146101)

Thornton, K., Bachtrog, D. \& Andolfatto, P. 2006 X chromosomes and autosomes evolve at similar rates in Drosophila: no evidence for faster-X protein evolution. Genome Res. 16, 498-504. (doi:10.1101/gr. 4447906)

Vicoso, B. \& Charlesworth, B. 2006 Evolution on the X chromosome: unusual patterns and processes. Nat. Rev. Genet. 7, 645-653. (doi:10.1038/nrg1914)

Vicoso, B. \& Charlesworth, B. 2009 Effective population size and the faster-X effect: an extended model. Evolution 63, 2413-2426. (doi:10.1111/j.1558-5646.2009. 00719.x)

Vicoso, B., Haddrill, P. R. \& Charlesworth, B. 2008 A multispecies approach for comparing sequence evolution of $\mathrm{X}$-linked and autosomal sites in Drosophila. Genet. Res. Camb. 90, 421-431. (doi:10.1017/S0016672308009804)

Zeyl, C., Vanderford, T. \& Carter, M. 2003 An evolutionary advantage of haploidy in large yeast populations. Science 299, 555-558. (doi:10.1126/science.1078417) 